\title{
Chemical recycling of polyhydroxybutyrate (PHB) into bio-based solvents and their use in a circular PHB extraction
}

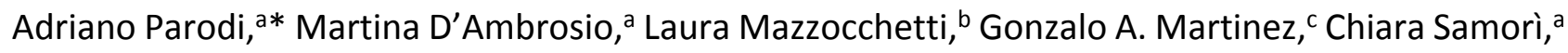
Cristian Torri, ${ }^{a}$ Paola Gallettia

Correspondence to: adriano.parodi2@unibo.it

\footnotetext{
a Dipartimento di Chimica "Giacomo Ciamician", University of Bologna, via Sant'Alberto 163, Ravenna, Italy

b Dipartimento di Chimica Industriale "Toso Montanari", University of Bologna, viale del Risorgimento 4, Bologna, Italy

c Dipartimento di Ingegneria Civile, Chimica, Ambientale e dei Materiali, University of Bologna, viale Risorgimento 2, Bologna, Italy
}

Number of pages : 13

Number of figures : 7

Number of tables : 6

\section{Table of contents}

1. MHB and MMB synthesis and screening conditions p. S2

$\begin{array}{ll}\text { 2. MHB and MMB biodegradability test } & \text { p. S6 }\end{array}$

$\begin{array}{ll}\text { 3. } \mathrm{MHB} \text { and } \mathrm{MMB} \text { recyclability test } & \text { p. S7 }\end{array}$

$\begin{array}{ll}\text { 4. Characterization of MHB, MC and MMB } & \text { p. S9 }\end{array}$

5. ${ }^{1} \mathrm{H}-\mathrm{NMR}$ and ${ }^{13} \mathrm{C}-\mathrm{NMR}$ Spectra of MHB, MC and MMB

$\begin{array}{ll}\text { 6. References } & \text { p. } \mathbf{S 1 3}\end{array}$ 


\section{MHB and MMB synthesis and screening conditions}

\section{MHB from pure PHB and PHB inclusions}

$\mathrm{PHB}, \mathrm{MeOH}$ and catalyst in different molar ratio were charged in a closed cap glass reactor. The methanolysis reaction was carried out under different time and temperatures. After that, the reaction mixture was cooled to rt, an equimolar amount (respect to the catalyst) of $\mathrm{NaOH}$ (in the case of $\mathrm{H}_{2} \mathrm{SO}_{4}$ and $\mathrm{TsOH}$ ) or $\mathrm{HCl}$ (in the case of $\mathrm{NaOH}$ and $\mathrm{NaOMe}$ ) was added and the solution was stirred at $\mathrm{rt}$ for $15 \mathrm{~min}$. The resulting solution was distilled to recover the unreacted methanol (room pressure) and $\mathrm{HMB}\left(75-80^{\circ} \mathrm{C}\right.$ under reduced pressure of 10 mbar).

Table S1. Optimization of reaction conditions for MHB synthesis from PHB.

\begin{tabular}{|c|c|c|c|c|c|c|}
\hline Substrate & Catalyst & $\begin{array}{l}\text { Cat. loading } \\
\text { (mol\%) }\end{array}$ & $\begin{array}{l}\mathrm{MeOH} \\
\text { (eq.) }\end{array}$ & $\mathrm{T}\left({ }^{\circ} \mathrm{C}\right)^{\mathrm{a}}$ & Time (h) & Yield (\%) \\
\hline pure $\mathrm{PHB}$ & $\mathrm{NaOMe}$ & 1 & 20 & 140 & 4 & 4 \\
\hline pure $\mathrm{PHB}$ & $\mathrm{CaO}$ & 1 & 20 & 140 & 4 & 7 \\
\hline pure $\mathrm{PHB}$ & $\mathrm{SnCl}_{2}$ & 1 & 20 & 140 & 4 & 0 \\
\hline pure $\mathrm{PHB}$ & $\mathrm{TsOH}$ & 1 & 20 & 140 & 4 & 77 \\
\hline pure $\mathrm{PHB}$ & $\mathrm{Sn}(\mathrm{Bu})_{2}(\mathrm{OAc})_{2}$ & 1 & 20 & 140 & 4 & 85 \\
\hline pure PHB & $\mathrm{H}_{2} \mathrm{SO}_{4}$ & 1 & 20 & 140 & 4 & 88 \\
\hline pure $\mathrm{PHB}$ & $\mathrm{H}_{2} \mathrm{SO}_{4}$ & 1 & 20 & 140 & 6.5 & 98 \\
\hline pure $\mathrm{PHB}$ & $\mathrm{H}_{2} \mathrm{SO}_{4}$ & 1 & 10 & 140 & 7 & 81 \\
\hline pure $\mathrm{PHB}$ & $\mathrm{H}_{2} \mathrm{SO}_{4}$ & 1 & 5 & 140 & 7 & 63 \\
\hline pure $\mathrm{PHB}$ & $\mathrm{H}_{2} \mathrm{SO}_{4}$ & 0.5 & 15 & 140 & 7 & 95 \\
\hline pure $\mathrm{PHB}$ & $\mathrm{H}_{2} \mathrm{SO}_{4}$ & 0.25 & 15 & 140 & 7 & 61 \\
\hline pure PHB & $\mathrm{Sn}(\mathrm{Bu})_{2}(\mathrm{OAc})_{2}$ & 0.5 & 15 & 140 & 7 & 87 \\
\hline pure $\mathrm{PHB}$ & $\mathrm{TsOH}$ & 0.5 & 15 & 140 & 7 & 79 \\
\hline pure $\mathrm{PHB}$ & $\mathrm{H}_{2} \mathrm{SO}_{4}$ & 0.5 & 15 & 70 & 7 & 3 \\
\hline SSC-57 & $\mathrm{H}_{2} \mathrm{SO}_{4}$ & 0.5 & 15 & 140 & 7 & $10^{\mathrm{b}}$ \\
\hline SSC-57 & $\mathrm{H}_{2} \mathrm{SO}_{4}$ & 5 & 15 & 140 & 7 & $53^{b}$ \\
\hline SSC-57 & $\mathrm{H}_{2} \mathrm{SO}_{4}$ & 10 & 15 & 140 & 7 & $85^{b}$ \\
\hline
\end{tabular}

a reaction conducted at $140{ }^{\circ} \mathrm{C}$ under autogenous pressure ${ }^{b}$ Yields are calculated respect to the $\mathrm{PHB}$ content inside bacterial cells. 


\section{$\mathrm{H}_{2} \mathrm{SO}_{4}$ recycle in MHB synthesis}

To demonstrate the recyclability of the acidic catalyst $\left(\mathrm{H}_{2} \mathrm{SO}_{4}\right)$, the reaction mixture was not quenched with $\mathrm{NaOH}$ at the end of the reaction: the solution was distilled to remove $\mathrm{MeOH}$ and then $\mathrm{MHB}$, the distillation residue and the recovered $\mathrm{MeOH}$ were directly reused to re-perform methanolysis on PHB.

Table S2. Catalyst recycle in MHB synthesis.

\begin{tabular}{|c|c|c|c|}
\hline Cycle & $\begin{array}{c}\text { MHB yield (mass, g; } \\
\text { Yield, \%)a }\end{array}$ & $\begin{array}{c}\text { MeOH recovery (mass, } \mathrm{g} \text {; } \\
\text { Yield, \%) }\end{array}$ & $\begin{array}{c}\text { Distillation Residue } \\
\text { (mass, g) }\end{array}$ \\
\hline 1 & $2.8 ; 82$ & $12.8 ; 98.3$ & 0.58 \\
\hline II & $3.1 ; 91$ & $12.95 ; 99.5$ & 0.70 \\
\hline III & $3.15 ; 93$ & $12.85 ; 98.7$ & 0.82 \\
\hline IV & $3.23 ; 95$ & $13 ; 99.8$ & 0.90 \\
\hline $\mathrm{V}$ & $3.28 ; 96$ & $12.95 ; 99.5$ & 0.95 \\
\hline
\end{tabular}

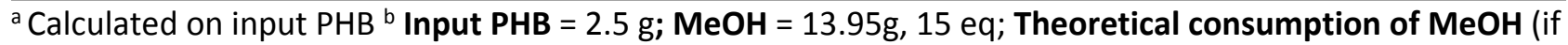
$100 \% \mathrm{MHB}$ yield) = $930 \mathrm{mg}$; recoverable $\mathrm{MeOH}$ (if 100\% $\mathrm{MHB}$ yield) $=13.02 \mathrm{~g}$ 
MMB from pure PHB and PHB inclusions

\section{Synthesis of MC}

Previously synthesized $\mathrm{CA},{ }^{1}$ heterogeneous acidic catalyst ( $w t \%$ respect to $\mathrm{CA}$ ) and $\mathrm{MeOH}$ in different molar ratios were charged in a closed cap glass reactor. The esterification reaction was carried out under different times and temperatures. After that, the solution was filtrated to recover the heterogeneous catalyst. The filtrate was distilled using a Vigreux condenser to recover, firstly unreacted methanol mixed with water (produced during the reaction) at atmospheric pressure, and then $\mathrm{MC}\left(\right.$ b.p. $\left.=118-120^{\circ} \mathrm{C}\right)$.

Table S3. Optimization of reaction conditions for MC synthesis from CA.

\begin{tabular}{|c|c|c|c|c|c|c|c|}
\hline Substrate & Catalyst & $\begin{array}{c}\text { Cat. } \\
\text { Loading } \\
\text { (wt \%) }\end{array}$ & $\begin{array}{c}\mathrm{MeOH} \\
\text { (eq) }\end{array}$ & $\mathrm{T}\left({ }^{\circ} \mathrm{C}\right)^{\mathrm{a}}$ & $\begin{array}{c}\text { Time } \\
\text { (h) }\end{array}$ & $\begin{array}{c}\text { GC-MS } \\
\text { conversion } \\
(\%)\end{array}$ & $\begin{array}{c}\text { Yield } \\
(\%)\end{array}$ \\
\hline $\mathrm{CA}$ & / & / & 20 & 130 & 5 & 20 & nd \\
\hline CA & $\begin{array}{c}\text { Amberlyst- } \\
15\end{array}$ & 10 & 15 & reflux & 24 & 50 & nd \\
\hline CA & $\begin{array}{c}\text { Amberlyst- } \\
15\end{array}$ & 10 & 20 & 130 & 5 & $>99$ & nd \\
\hline CA & $\begin{array}{c}\text { Amberlyst- } \\
15\end{array}$ & 10 & 10 & 130 & 5 & $>99$ & nd \\
\hline $\mathrm{CA}$ & $\begin{array}{c}\text { Amberlyst- } \\
15\end{array}$ & 5 & 20 & 130 & 5 & $>99$ & nd \\
\hline CA & $\begin{array}{c}\text { Amberlyst- } \\
15\end{array}$ & 5 & 10 & 130 & 5 & $>99$ & nd \\
\hline $\mathrm{CA}$ & $\begin{array}{c}\text { Amberlyst- } \\
15\end{array}$ & 2.5 & 10 & 130 & 5 & 98 & nd \\
\hline CA & $\begin{array}{c}\text { Amberlyst- } \\
15\end{array}$ & 5 & 5 & 130 & 5 & 97.5 & nd \\
\hline CA & $\begin{array}{c}\text { Amberlyst- } \\
15\end{array}$ & 2.5 & 5 & 130 & 5 & 94 & 84 \\
\hline CA & $\begin{array}{c}\text { Amberlyst- } \\
15\end{array}$ & 2.5 & 5 & 130 & 7 & 98 & 89 \\
\hline $\mathrm{CA}$ & $\mathrm{C}-\mathrm{SO}_{3} \mathrm{H}^{\mathrm{b}}$ & 2.5 & 5 & 130 & 5 & 96 & 88 \\
\hline $\mathrm{CA}$ & $\mathrm{C}-\mathrm{SO}_{3} \mathrm{H}$ & 2.5 & 5 & 130 & 7 & $>99$ & 93 \\
\hline CA (SSC-57) & $\mathrm{C}-\mathrm{SO}_{3} \mathrm{H}$ & 2.5 & 5 & 130 & 7 & $>99$ & 91 \\
\hline
\end{tabular}

a reaction conducted at $130{ }^{\circ} \mathrm{C}$ under autogenous pressure $.{ }^{b} \mathrm{C}-\mathrm{SO}_{3}$ synthesized following a previously reported procedure. ${ }^{2}$ 


\section{Synthesis of MMB}

$\mathrm{MeOH}$ and catalyst were mixed under magnetic stirring in a round bottomed flask at $\mathrm{rt}$ for $15 \mathrm{~min}$, then $\mathrm{MC}$ was added dropwise over 30 minutes and the solution stirred at different times and temperatures. After that time, the resulting solution was distilled to recover $\mathrm{MeOH}$, unreacted $\mathrm{MC}$ and $\mathrm{MMB}\left(65-70{ }^{\circ} \mathrm{C}\right.$ under reduced pressure of $10 \mathrm{mbar}$ ).

Table S4. Optimization of reaction conditions for MMB synthesis from MC.

\begin{tabular}{|c|c|c|c|c|c|c|c|}
\hline Substrate & Catalyst & $\begin{array}{c}\text { Cat. } \\
\text { Loading } \\
\text { (\%) }\end{array}$ & $\begin{array}{c}\mathrm{MeOH} \\
(\mathrm{eq})\end{array}$ & $\mathrm{T}\left({ }^{\circ} \mathrm{C}\right)$ & Time (h) & $\begin{array}{c}\text { GC-MS } \\
\text { conversion } \\
(\%)\end{array}$ & Yield (\%) \\
\hline $\mathrm{MC}$ & $\mathrm{ZnCl}_{2}$ & 10 & 2.5 & r.t. & 24 & 0 & nd \\
\hline$M C$ & $\mathrm{Nal}$ & 10 & 2.5 & r.t. & 24 & 0 & nd \\
\hline$M C$ & Morpholine & 10 & 2.5 & r.t. & 24 & 0 & nd \\
\hline$M C$ & DBU & 10 & 2.5 & r.t. & 24 & 46 & nd \\
\hline$M C$ & DMCA & 10 & 2.5 & r.t. & 24 & 0 & nd \\
\hline$M C$ & $\mathrm{CaO}$ & 10 & 2.5 & r.t. & 24 & 3 & nd \\
\hline$M C$ & $\mathrm{CuO}$ & 10 & 2.5 & r.t. & 24 & 0 & nd \\
\hline $\mathrm{MC}$ & $\mathrm{PBu}_{3}$ & 10 & 2.5 & r.t. & 24 & 17 & nd \\
\hline$M C$ & $\mathrm{Na}_{2} \mathrm{CO}_{3}$ & 10 & 2.5 & r.t. & 24 & 0 & nd \\
\hline$M C$ & $\mathrm{~K}_{2} \mathrm{CO}_{3}$ & 10 & 2.5 & r.t. & 24 & 82 & nd \\
\hline$M C$ & $\mathrm{NaOH}$ & 10 & 2.5 & r.t. & 24 & 48 & nd \\
\hline$M C$ & $\mathrm{KOH}$ & 10 & 2.5 & r.t. & 24 & 42 & nd \\
\hline$M C$ & $\mathrm{NaOMe}$ & 10 & 10 & r.t. & 16 & 95 & 90 \\
\hline$M C$ & $\mathrm{NaOMe}$ & 10 & 2.5 & r.t. & 24 & 98 & 93 \\
\hline $\mathrm{MC}$ & $\mathrm{NaOMe}$ & 10 & 2.5 & 60 & 24 & 87 & nd \\
\hline $\mathrm{MC}$ & $\mathrm{NaOtBu}$ & 10 & 2.5 & r.t. & 24 & 97 & 94 \\
\hline$M C$ & $\mathrm{NaOMe}$ & 5 & 2.5 & r.t. & 24 & 90 & nd \\
\hline$M C$ & $\mathrm{NaOMe}$ & 5 & 2.5 & r.t. & 50 & 98 & nd \\
\hline$M C$ & $\mathrm{NaOMe}$ & 3 & 2 & r.t. & 50 & 98 & 92 \\
\hline$M C$ & $\mathrm{NaOMe}$ & 1.5 & 2 & r.t. & 50 & 85 & nd \\
\hline MC (SSC-57) & $\mathrm{NaOMe}$ & 3 & 2 & r.t. & 50 & 95 & 90 \\
\hline
\end{tabular}




\section{MHB and MMB biodegradability test}

Biodegradation test of $\mathrm{HMB}$ and MMB were conducted according to OECD guideline $301 \mathrm{f.}^{3}$

Figure S1. MHB and MMB biodegradation over 28 days.

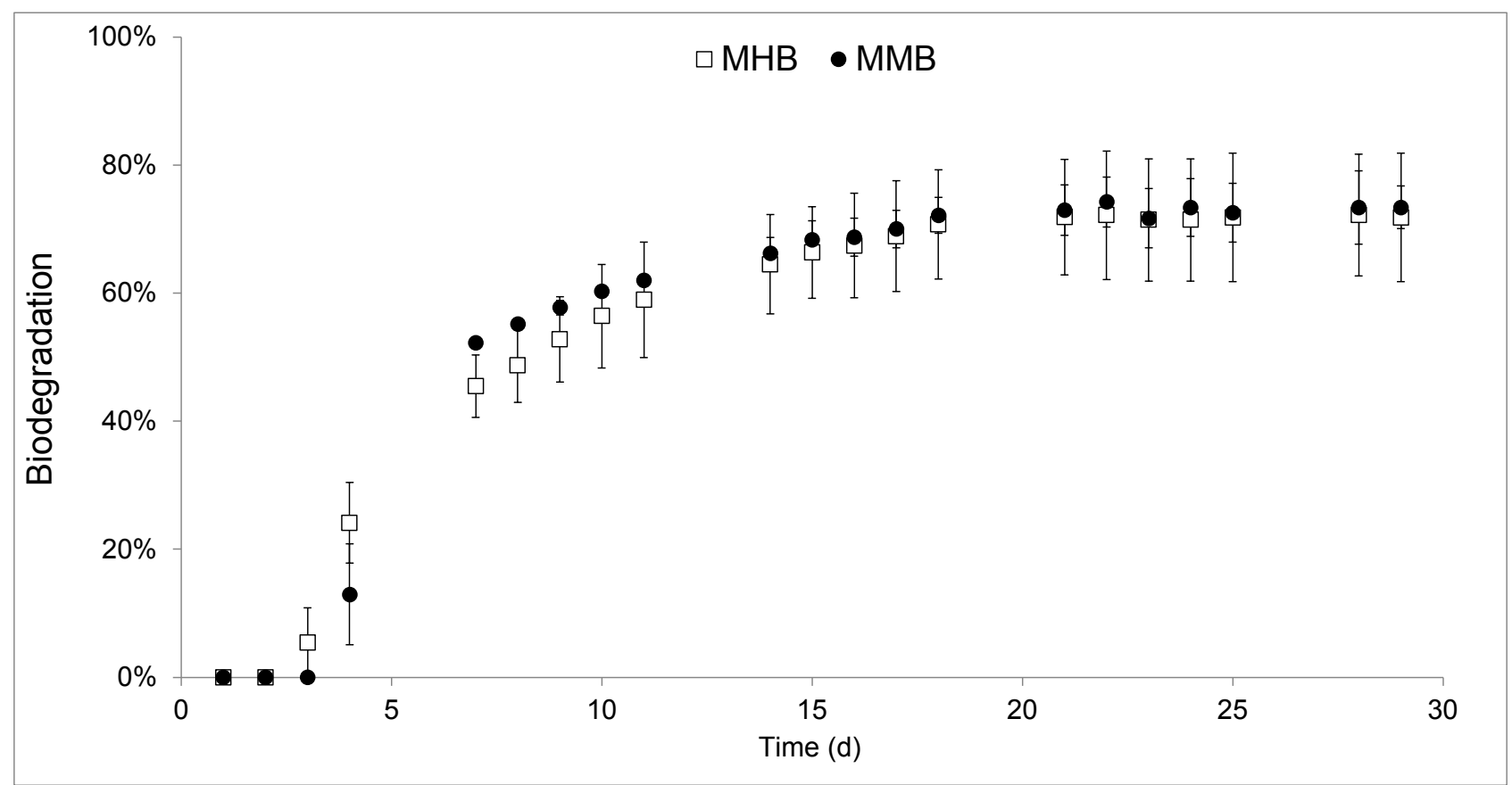




\section{MHB and MMB recyclability test}

Table S5. MMB extraction recycle (PHB-to-solvent ratio $=26 \mathrm{mg} / \mathrm{mL}$; Volume tested $=5 \mathrm{~mL}$ ).

\begin{tabular}{ccc}
\hline Cycle & PHB recovery (\%) & Solvent recovery (\%) \\
& & \\
\hline 1 & 99 & 100 \\
2 & 96 & 95 \\
3 & 98 & 98 \\
4 & 99 & 99 \\
5 & 96 & 98 \\
6 & 99 & 99 \\
7 & 98 & 96 \\
8 & 99 & 97 \\
9 & 95 & 96 \\
10 & 99 & 100 \\
11 & 99 & 99 \\
12 & 96 & 95 \\
\hline
\end{tabular}

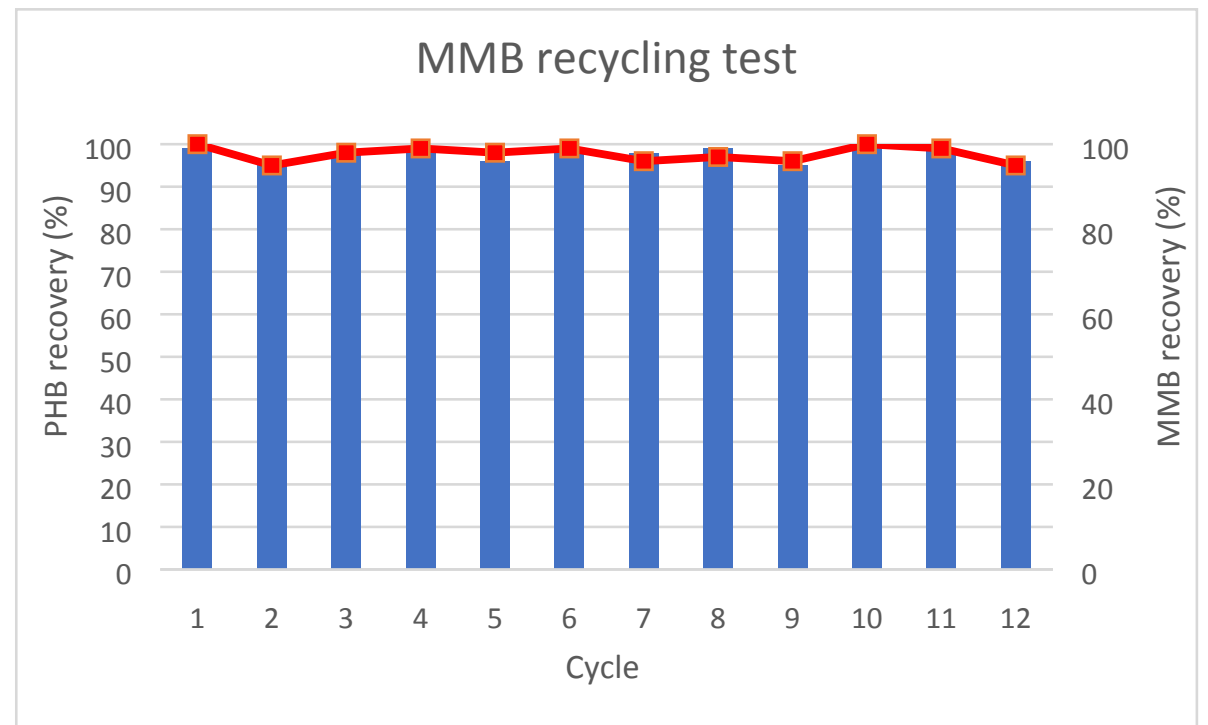


Table S6. MHB extraction recycle (PHB-to-solvent ratio $=26 \mathrm{mg} / \mathrm{mL}$; Volume tested $=5 \mathrm{~mL}$ ).

\begin{tabular}{ccc}
\hline Cycle & PHB recovery (\%) & Solvent recovery (\%) \\
& & \\
\hline 1 & 95 & 91 \\
2 & 94 & 88 \\
3 & 97 & 89 \\
4 & 93 & 87 \\
5 & 94 & 85 \\
6 & 97 & 88 \\
7 & 98 & 90 \\
8 & 94 & 91 \\
9 & 95 & 88 \\
10 & 94 & 89 \\
11 & 97 & 90 \\
12 & 96 & 88 \\
\hline
\end{tabular}

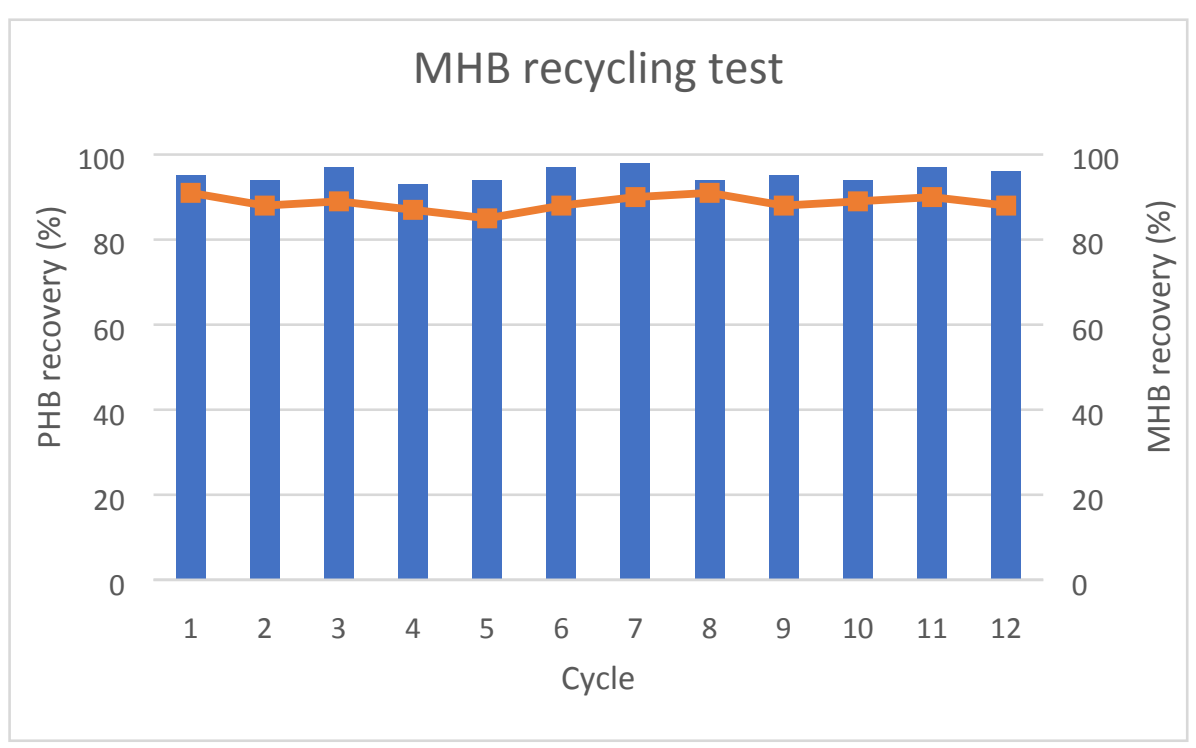




\section{Characterization of synthesized products}

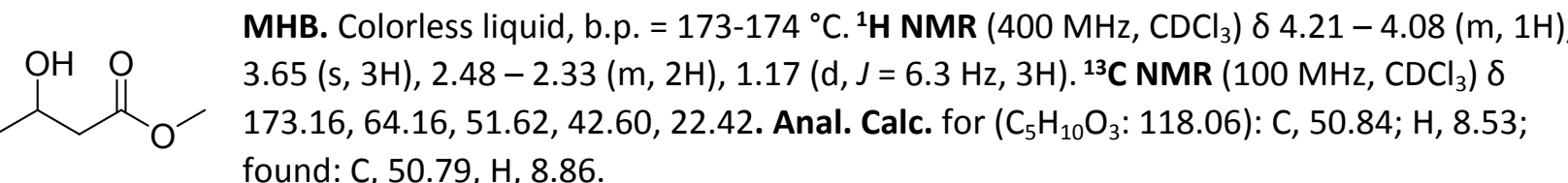

found: C, $50.79, \mathrm{H}, 8.86$.<smiles>C/C=C/C(=O)OC</smiles>

MC. Colorless liquid, b.p. $=118-120^{\circ} \mathrm{C} .{ }^{1} \mathrm{H}$ NMR $\left(400 \mathrm{MHz}, \mathrm{CDCl}_{3}\right) \delta 6.96(\mathrm{dq}, J=15.5,6.9$ $\mathrm{Hz}, 1 \mathrm{H}), 5.83(\mathrm{dq}, J=15.6,1.7 \mathrm{~Hz}, 1 \mathrm{H}), 3.70(\mathrm{~s}, 3 \mathrm{H}), 1.86(\mathrm{dd}, J=6.9,1.7 \mathrm{~Hz}, 3 \mathrm{H}) .{ }^{13} \mathrm{C}$ NMR $\left(100 \mathrm{MHz}, \mathrm{CDCl}_{3}\right) \delta 166.98,144.72,122.35,51.33,17.94$.

MMB. Colorless liquid, b.p. $=148-149^{\circ} \mathrm{C} .{ }^{1} \mathrm{H}$ NMR $\left(401 \mathrm{MHz}, \mathrm{CDCl}_{3}\right) \delta 3.77-3.67(\mathrm{~m}$,<smiles>COC(=O)CC(C)OC</smiles>
$1 \mathrm{H}), 3.64(\mathrm{~s}, 3 \mathrm{H}), 3.29(\mathrm{~s}, 3 \mathrm{H}), 2.53(\mathrm{dd}, J=15.1,7.2 \mathrm{~Hz}, 1 \mathrm{H}), 2.33(\mathrm{dd}, J=15.1,5.7 \mathrm{~Hz}$, $1 \mathrm{H}), 1.16(\mathrm{~d}, J=6.2 \mathrm{~Hz}, 3 \mathrm{H}) .{ }^{13} \mathrm{C}$ NMR $\left(100 \mathrm{MHz}, \mathrm{CDCl}_{3}\right) \delta 171.88,73.51,56.26,51.52$, 41.43, 19.10. Anal. Calc. for $\left(\mathrm{C}_{6} \mathrm{H}_{12} \mathrm{O}_{3}\right.$ : 118.06): $\mathrm{C}, 54.53 ; \mathrm{H}, 9.15$; found: $\mathrm{C}, 54.55, \mathrm{H}, 9.20$. 


\section{5. ${ }^{1} \mathrm{H}-\mathrm{NMR}$ and ${ }^{13} \mathrm{C}$-NMR Spectra of MHB, MC and MMB}

Figures S2 and S3. ${ }^{1} \mathrm{H}-\mathrm{NMR}$ (up) and ${ }^{13} \mathrm{C}-\mathrm{NMR}$ (down) of MHB.
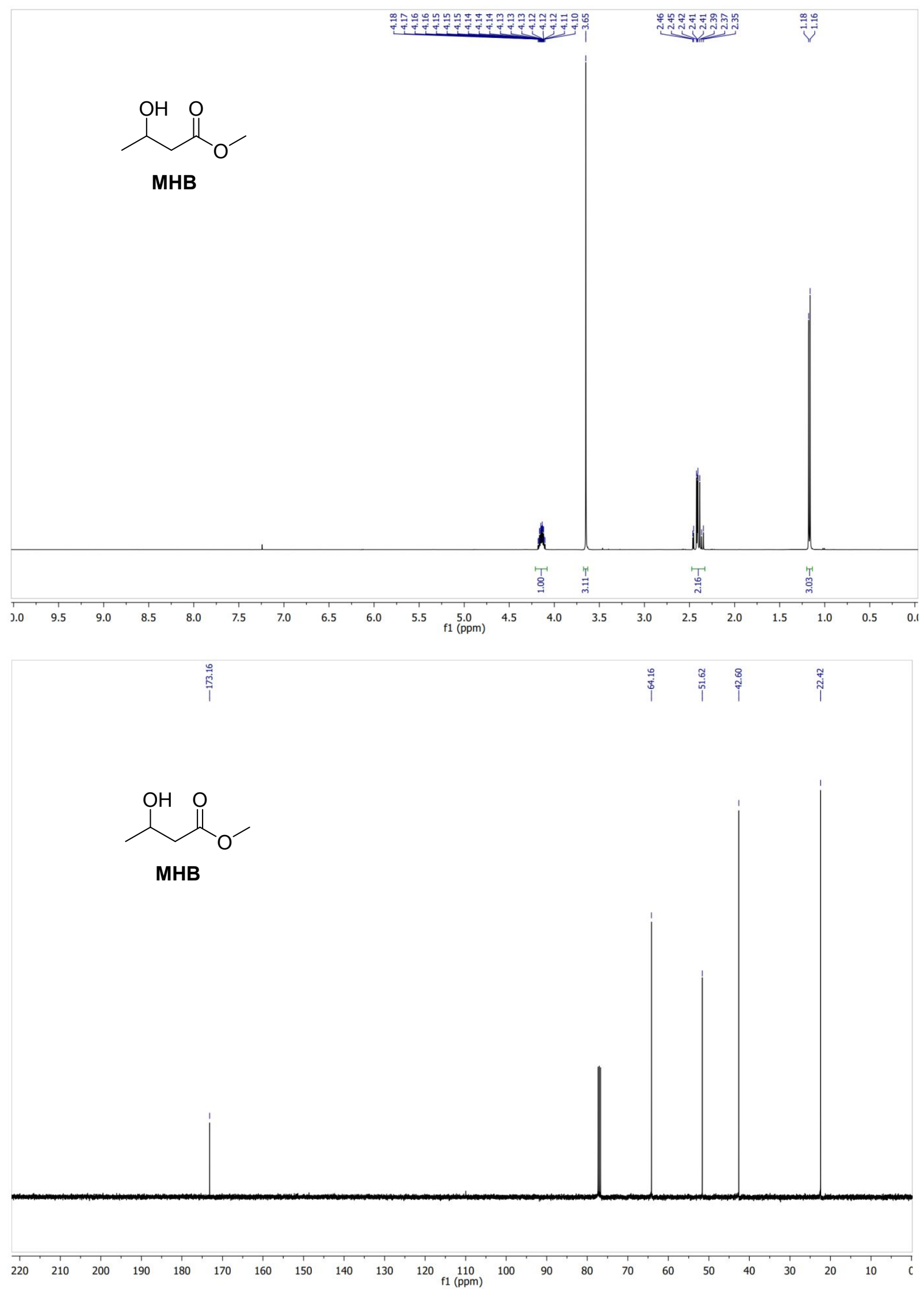
Figures S4 and S5. ${ }^{1} \mathrm{H}-\mathrm{NMR}$ (up) and ${ }^{13} \mathrm{C}-\mathrm{NMR}$ (down) of MC.
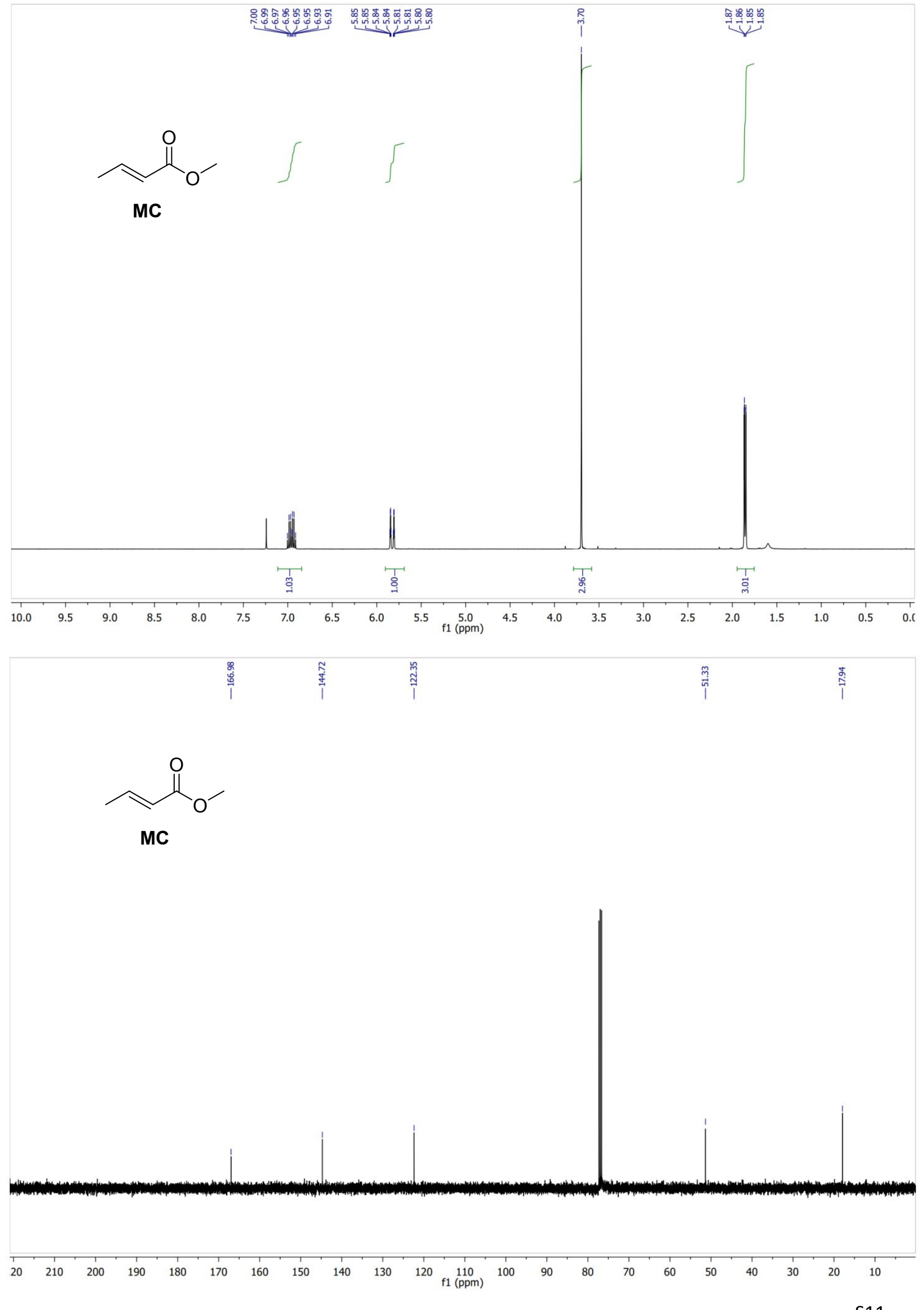
Figures $\mathrm{S} 6$ and $\mathrm{S} 7 .{ }^{1} \mathrm{H}-\mathrm{NMR}$ (up) and ${ }^{13} \mathrm{C}-\mathrm{NMR}$ (down) of MMB.
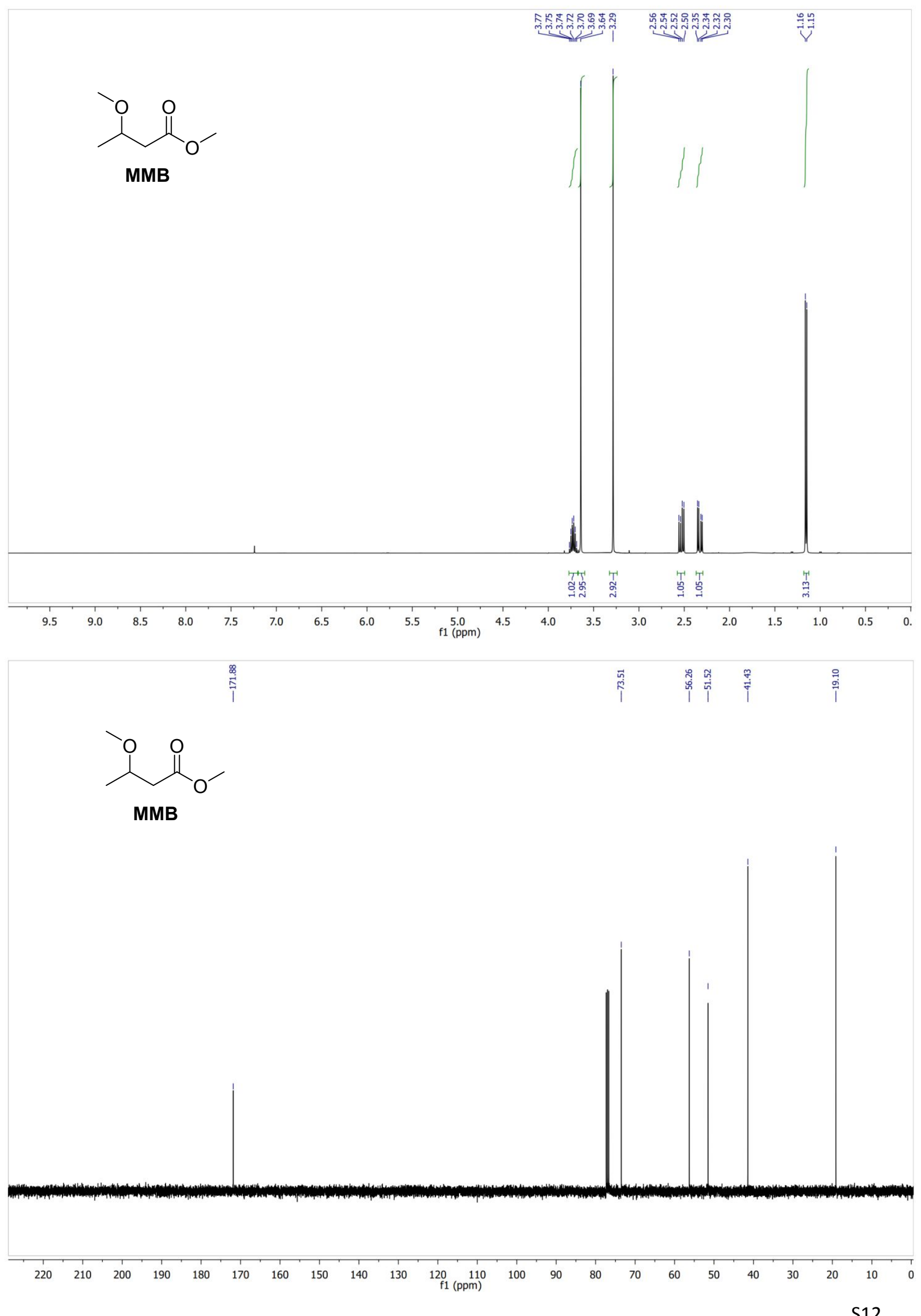


\section{References}

1 A. Parodi, A. Jorea, M. Fagnoni, D. Ravelli, C. Samori, C. Torri and P. Galletti, Green Chem., 2021, 3420-3427.

2 C. Samorì, A. Parodi, E. Tagliavini and P. Galletti, J. Anal. Appl. Pyrolysis, 2021, 155, art. $\mathrm{n}^{\circ}$ 105030.

3 OECD, OECD Guidel. Test. Chem., 1992, 301, 1-62. 